\title{
Spinal Cord Mechanics During Flexion and Extension of the Cervical Spine: A Finite Element Study
}

\author{
Jeffrey Scifert, PhD*, Koji Totoribe, MD**, Vijay Goel, PhD\# and Jan Huntzinger, BS*
}

The cervical spinal cord is at a great risk for injury during traumatic accidents occurring within people of relatively young age. Spinal cord injury can not be easily characterized clinically nor through in vitro testing. An understanding of cervical spinal cord deformation is important for defining an injury threshold of the spinal cord. Therefore, an analytical method may be beneficial for determining the response of the spinal cord response to external loads on the spine.

We have developed a three-dimensional, non-linear finite element model to quantify physiologic strains and stresses in the cervical spinal cord placed in the ligamentous C5-C6

Several spinal disorders and traumatic loading situations are known to inflict damage to neurovascular components of the cervical spinal cord. The spinal cord can be cut in an accident, compressed, destroyed by infection, damaged when its blood supply is cut off, or affected by diseases that alter its nerve root function. Economically, physically, and socially, spinal cord trauma is a debilitating occurrence. Around $56 \%$ of spinal cord injuries occur in people under the age of 30 , with the highest incidence rates in people 16-30 years of age, a youthful and otherwise healthy population. A percentage breakdown of the causes of spinal cord trauma is as follows: $37 \%$ due to motor vehicle accidents, specifically whiplash injuries, $21 \%$ due to falls, $6 \%$ due to sports (particularly diving-related

From Medtronic Sofamor Danek, Memphis, TN; Department of Orthopedic Surgery, Miyazaki Medical College, Japan; and Spine Research Center, University of Toledo, and Medical College of Ohio, Toledo, OH. *Dr. Scifert is Product development engineer at Medtronic Sofamor Danek, **Dr. Totoribe is Associate Professor at Department of Orthopedic Surgery, ${ }^{\#}$ Dr. Goel is Professor and Chairman and Ms. Huntzinger is Graduate student at University of Toledo, $\mathrm{OH}$. Address correspondence: Vijay Goel, PhD, Department of Bioengineering, College of Engineering, NI5051C, University of Toledo, Toledo, $\mathrm{OH}$ 43606. E-mail: vijay.goel@utoledo.edu

Acknowledgement: Work supported in part by a grant from the Cervical Spine Research Society. motion segment. Compressive loads along with flexion and extension moments were applied to the bony segment and not the cord itself. Motion data, shown in degrees of movement in flexion and extension calculated for the model showed a close representation to in vivo data. Von Mises stress plots and strains on the cervical spinal cord were computed. This model represents a first attempt by the authors to analytically quantify the mechanical response of the spinal cord to cervical spine injury.

Keywords: Spinal cord, cervical spine, flexion, extension, whiplash

injuries), and $28 \%$ due to acts of violence (i.e., firearms). Though the number of injuries resulting from vehicular accidents is decreasing, the injuries due to violence are rapidly increasing.

The incidence of spinal cord injury, as recorded by the highest level of injury, occurs in the upper cervical (C1$\mathrm{C} 2$ ) region in $11 \%$, the lower cervical (C3-C7) in 51\%, the upper thoracic (T1-T10) in 14\%, the thoracolumbar (T11-L2) in $20 \%$ and the lumbar/lumbosacral (L3-S3) segment in $4 \%$ of patients(1). Clearly the lower cervical spine is at the greatest risk of traumatic injury of the spine and spinal cord; whiplash being a common source of lower cervical spine injury.

The precise mechanism of whiplash is an uncertain issue. Theories range from whiplash being defined as a hyperextension-type injury to being caused by posterior hypertranslation of the head (2-5). The severity of whiplash trauma, as documented from radiographs, does not correlate with the seriousness of clinical problems such as pain in the neck and headache. This suggests that radiographs alone do not adequately describe the whiplash mechanism.

Clinical disorders observed in whiplash have been relatively well documented. However, clinical investigations have shown no apparent relation between 
the degree of neurologic deficit and canal occlusion $(6,7)$. This apparent discrepancy may be due to significant differences between canal height and mid-sagittal diameter during and immediately following impact as compared to these dimensions measured long after impact (for instance, 2 hours post-impact) (8-10). Through in vitro experiments, investigators have found that post-injury $65 \%$ of the maximum canal height loss and $269 \%$ of the mid-sagittal diameter occlusion, that had occurred under the traumatic compressive load, are recovered. Thus, post-injury geometry is not useful in predicting transient canal geometry, indicating the severity of injury can be masked by the clinical presentation of canal geometry found postinjury. Likewise, by the time the patient is transported to the hospital and undergoes magnetic resonance imaging, the cord and associated spinal structures have recovered compared to the state of immediate injury. Thus, a true diagnosis of the pathomechanism of the injury is extremely difficult. Studies using in vitro techniques to observe these cervical spinal and cord changes would not be feasible due to the changes in the spinal cord after dissection as well as to changes in spinal geometry compared to at the time of an injury.

In vitro cadaveric studies are conducted in an attempt to simulate whiplash injury as cord deformations have been seen in a flexion-compression model of the upper thoracic spine. However, it is difficult to reproduce the in vivo environment in order to quantify changes in the spinal cord deformation and structure.

An understanding of physiologic cervical spinal cord deformation is important for defining an injury threshold of the cervical spinal cord. Studies have shown that damage to the spinal cord can occur regardless of significant damage to surrounding structures.

The shape and size of the structures seen on radiographs and MRI taken long after the trauma do not correlate well with clinical symptoms such as pain. Since the spinal column undergoes rapid compression and then recovery during and after impact loads, the stresses, strains, and deformations in the spinal cord, spinal ligaments and bony structures will decrease by the time a patient is scanned for possible lesions. There is no data correlating the behavior of the human spinal cord during impact delivered to the head with the clinical symptoms in vivo. Thus, although there is a need to undertake in vivo studies on humans during impact, such investigations are not practical. Likewise, animal studies are not practical as the forces generated during a whiplash injury cannot be adequately represented with a quadruped. Similarly, in vitro tests cannot address this issue.

Most of the relevant research to date has been performed experimentally with many strict restrictions and thus the analytical methods proposed here may possibly overcome these restraints. Analytical methods have been widely applied in spinal problems to quantify the changes of each spinal element, though biomechanical aspects of spinal cord injury have not been thoroughly investigated. Finite element analysis is the most popular analytical method for quantifying stresses in the spinal column. In this research, a three-dimensional finite element model of C56 motion segment including dural-sac element and the spinal cord has been developed to investigate the effects of mechanical forces on the spinal cord. At the time of injury, the spinal cord is subjected to different combinations of shear and compressive forces in addition to possible distraction. To understand the mechanics of spinal cord injury, one needs to quantify stresses and strains within the spinal cord in response to external loads applied to the bony spine. As has been previously discussed, experimental studies cannot address this issue. However, we hypothesize that an analytical method may be able to quantify the forces in the cervical spinal column to predict their effects on the spinal cord.

\section{METHODS}

Computerized tomography (CT) scans, anatomic textbooks, and other information from the literature were used to develop the three-dimensional non-linear ligamentous model, an approach used in our earlier finite element (FE) models (11). A 'normal' fresh frozen human ligamentous cervical spine was scanned at $1.0 \mathrm{~mm}$ intervals along the lower cervical spine (C3-C7). Radiographs and DEXA (dual-energy x-ray absorptometry) were used to ensure no osseous abnormalities existed with the specimen and that the bone quality was good. The lower cervical region from mid-C2 to mid-T1 was scanned (settings: $120 \mathrm{kV}, 200 \mathrm{~mA}, 1 \mathrm{sec}$ exposure time, slice thickness $=1.0$ $\mathrm{mm}$ ) using CT scan (Toshiba Xpress/SX ${ }^{\circledR}$, Tustin, CA). A total of 94 transverse plane scans were generated. The cross-sectional shapes of the bony geometry depicted in the transverse slices were divided into finite regions by creating a mesh structure by hand. Tracings were made of each of these images and points in each tracing were digitized to generate the FE model mesh using ABAQUS standard finite element package. The digitized sections were stacked serially in the appropriate order to obtain a three-dimensional model of the C5-C6 segment for the 


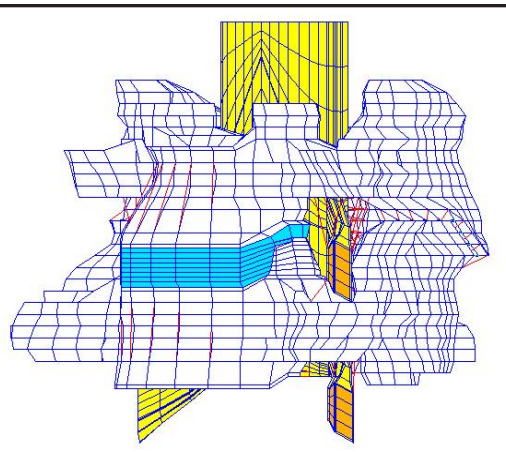

A.

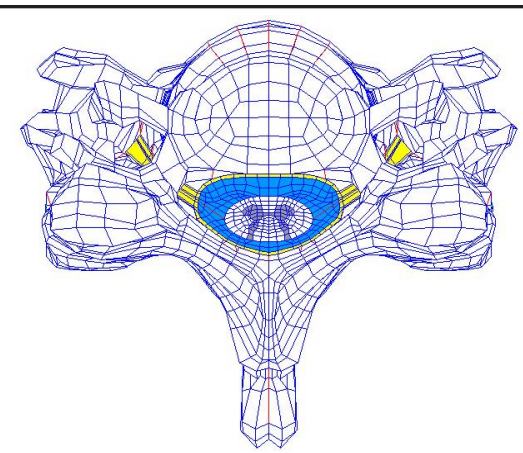

B.

Fig. 1. (A) Side view of C5-C6 spinal cord model with spinal cord and connective fibers. (B) Top view of the C5-C6 spinal cord model showing placement of spinal cord, CSF, and dural sheath in canal

purpose of this study (Figure 1). A $5^{\circ}$ segmental angular measure was used to create the lordotic curve for the $\mathrm{C} 5$ C6 model. This is within 1 standard deviation of the values obtained from the literature.

The spinal cord geometry was generated from pictures found in anatomical texts and atlases, (transverse slice pictures), pictures of fresh cadaver materials, and from published population data. The pictures were scanned and saved. The outline of the pertinent structures of the spinal cord (i.e. canal boundaries, grey and white matter, etc.), were "traced" using image processing utilities. These tracings were scaled to fit the canal dimensions in the finite element models described above. From these tracings, points were generated on the outlines and a mesh created. The mesh was built up layer by layer until a threedimensional model of the cord was generated (Figure 1). Changes in the cross-sectional area of the cord needed to be appropriately modeled as the area changes throughout the length of the cervical spine from $\mathrm{C} 0-\mathrm{C} 7$. Although a viscoelastic structure, the present model being quasi-static, cord material properties were assumed to have elastic properties based on values taken from the literature (12). (Table 1). Surface contact was simulated between the dural sheath of the cord and the vertebral elements in the spinal canal and posterior aspect of the $\mathrm{C} 5-\mathrm{C} 6$ intervertebral disc.

After modeling the main part of the spinal cord, the next step was to model the associated C5 and C6 spinal nerve roots, dentate ligaments, dural sheath, CSF, gray matter, white matter, connective fibers, and dura mater in a similar manner. The studies by Bilston et al. and Yuan et al. were helpful in providing us the material property data of the spinal cord for use in the model $(13,14)$. Also, studies involving human dura matter surrounding the brain were useful in simulating the dura matter of the spinal cord (15).
For some structures, material properties were approximated as those of similar biological materials due to lack of property definitions in the literature. The intact model contained 24,732 nodes and 21,895 elements (Figure 1).

The C6 inferior-most bony nodes and the bottom of the cord were fully constrained, while the top of the cord was left unconstrained. Surface contact was simulated between the dural sheath of the cord and the elements in the spinal canal and posterior aspect of the C5-C6 intervertebral disc. The global coordinate system of the model $(1,2$, and 3$)$ was oriented positive to the left looking posterior to anterior, positive anteriorly, and positive downward axially along the spine, respectively (Figure 1). All models were run using ABAQUS ${ }^{\circledR}$ (version 5.8, Hibbitt, Karlsson \& Sorenson, Inc., Pawtucket, RI). The model was subjected to pure axial compressive loading $(73.6 \mathrm{~N})$ with flexion or extension moments $(1.5 \mathrm{Nm})$ applied to the upper bony section of the $\mathrm{C} 5$ vertebra. Changes in cord and dura deformations/strains in the axial direction were computed. The resulting von Mises stress patterns and levels at the white/gray matter and the dural sheath of the cord were computed. The spinal cord strain $\left(\epsilon_{22}\right.$ strain, axial direction) values were computed from a set of elements along the anterior and posterior midlines of the cord ( 18 elements for $\mathrm{C} 5$ and 12 elements for C6). These elements were chosen because they lay behind the vertebral sections of the model. Similarly, the elements of the dural sheath chosen for analysis of the C5-C6 disc space also lay behind the disc along the anterior midline of the spinal cord.

\section{RESULTS}

Range of motion validation of the intact C5-C6 model was performed by comparing model predictions to published 
Table 1. Material Properties for the C5-C6 Model with Spinal Cord

\begin{tabular}{llll}
\hline Material & Element type & Young's Modulus, E (MPA) & Poisson's Ratio (v) \\
\hline Cotrical bone & 8-node solid & 10,000 & 0.3 \\
Cancellous bone & 8-node solid & 450 & 0.25 \\
Posterior bone & 8-node solid & 3,500 & 0.25 \\
Endplates & 8-node solid & 10,000 & 0.3 \\
Annulus Matrix & 8-node solid & 4.2 & 0.45 \\
Annulus Fibers & Rebar & 450 & 0.3 \\
Nucleus Pulposus & Fluid element & 1 & 0.499 \\
\hline Gray matter & 8-node solid & 0.0667 & 0.499 \\
White matter & 8-node solid & 0.0667 & 0.499 \\
Dural sheath & 8-node solid & 31.5 & 0.45 \\
CSF & 8-node solid & 0.001 & 0.499 \\
Spinal nerve & 8-node solid & 0.0667 & 0.499 \\
Nerve sheath & 8-node solid & 31.5 & 0.45 \\
\hline Spinal ligaments & & & \\
ALL & 2-node & $15(<12 \%) ; 30(>12 \%)$ & 0.3 \\
PLL & nonlinear & $10(<12 \%) ; 20(>12 \%)$ & 0.3 \\
CAP & tension & $7(<12 \%) ; 30(>12 \%)$ & 0.3 \\
LF & only & $5(<25 \%) ; 10(>25 \%)$ & 0.3 \\
ISL & cable elements & $4(20 \%-40 \%) ; 8(>40 \%)$ & 0.3 \\
\hline Denate ligament & 2-node tension & 10 & 0.3 \\
Connecting fibers & only cable element & 10 & 0.3 \\
\hline ALL & PLL & & 10.3 \\
\hline
\end{tabular}

$\overline{\mathrm{ALL}}=$ anterior longitudinal ligament, $\mathrm{PLL}=$ posterior longitudinal ligament, $\mathrm{LF}=$ ligamentum flavum, $\mathrm{ISL}=$ interspinous ligament, $\mathrm{CAP}=$ capsular ligaments

cadaveric load-displacement data. Load cases used for validation included $0.5,1.0$, or $1.5 \mathrm{Nm}$ moments in flexion/ extension, lateral bending, or axial rotation and a combined load of $73.6 \mathrm{~N}$ plus a $1.8 \mathrm{Nm}$ flexion/extension, lateral bending, or axial rotation moment. These loads correspond to loads applied in vitro by Onan et al. (16), Grauer et al (17), Clausen et al. (18), our own in vitro experiments (19), and Moroney et al.(20) Overall, predicted main and coupled rotations agreed well with the published in vitro data, typically within 1 or 2 standard deviations of experimental data (depending upon the study and level examined).

Intact motion segment model predictions demonstrated good agreement with in vitro data. The model predicted $3.5^{\circ}$ of flexion in response to the applied compressive and flexion moment. Assuming that each spinal segment contributes an equal amount of rotation, about $23^{\circ}$ of flexion will be needed across the $\mathrm{C} 0-\mathrm{T} 1$ level to produce $3.5^{\circ}$ of flexion at the $\mathrm{C} 5-\mathrm{C} 6$ level. In this case, the model predicted axial strains in the spinal cord were within the in vivo range corresponding to approximately $20^{\circ}$ of flexion reported in the literature (14) (Table 2). Average posterior and anterior axial strain values for the spinal cord white/gray matter in flexion were approximately $0.4-$ $1.0 \%$ less, respectively, at the $\mathrm{C} 5$ level than that described as the average result for the entire cervical spine at $20^{\circ}$ of flexion by Yuan et al. (14) (Table 2). The C6 average strain in the white/gray matter in flexion was less than the C5 level average strain (this effect may be partially due to 
Table 2. \% Strain in Cord and Dural Sheath During Flexion/Compression

\begin{tabular}{|c|c|c|c|c|}
\hline & $\begin{array}{l}\text { Spinal cord- } \\
\text { flexion }\end{array}$ & $\begin{array}{l}\text { Dural sheath- } \\
\text { flexion }\end{array}$ & $\begin{array}{l}\text { Spinal cord- } \\
\text { extension }\end{array}$ & $\begin{array}{l}\text { Dural sheath- } \\
\text { extension }\end{array}$ \\
\hline $\begin{array}{l}\text { Average anterior \% strain @20 deg. flexion(whole cord - Yuan } \\
\text { et al.) }\end{array}$ & $\sim 2.3 \%$ & NA & NA & NA \\
\hline Average anterior \% strain(C5 vertebra -current model) & $1.3 \%$ & $1.3 \%$ & $-0.8 \%$ & $-2.2 \%$ \\
\hline Average anterior \% strain(C6 vertebra - current model) & $0.1 \%$ & $0.5 \%$ & $0.9 \%$ & $-0.5 \%$ \\
\hline Average anterior \% strain(C5-C6 disc space - current model) & NA & $3.8 \%$ & NA & $-3.1 \%$ \\
\hline $\begin{array}{l}\text { Average posterior \% strain @ 20deg. flexion(whole cord - } \\
\text { Yuan et al.)(14) }\end{array}$ & $\sim 2.6 \%$ & NA & NA & NA \\
\hline Average posterior \% strain(C5 vertebra - current model) & $2.1 \%$ & $5.4 \%$ & $-0.5 \%$ & $-4.0 \%$ \\
\hline Average posterior \% strain(C6 vertebra - current model) & $1.1 \%$ & $1.2 \%$ & $-0.7 \%$ & $-1.2 \%$ \\
\hline
\end{tabular}

boundary conditions imposed at the inferior end of the cord). The strain in the elements of the dural sheath directly behind the disc evidenced an increased average strain compared to the average strain of the $\mathrm{C} 5$ level behind the bony vertebra for both flexion and extension, due to contact between the disc and dural sheath. Von Mises stress patterns indicate higher stresses anteriorly and posteriorly during flexion and extension, particularly on the anterior side of the dural sheath where it contacts the disc during flexion (disc bulge) (Figure 2).

\section{DISCUSSION}

The trend of the strain data indicates that the posterior surface of the cord was strained more than the anterior surface during flexion, which is in agreement with the data from Yuan et al. Also, the von Mises stress plots indicate an increase in stress on the dural sheath during flexion due to disc bulging. Since our data on predicted

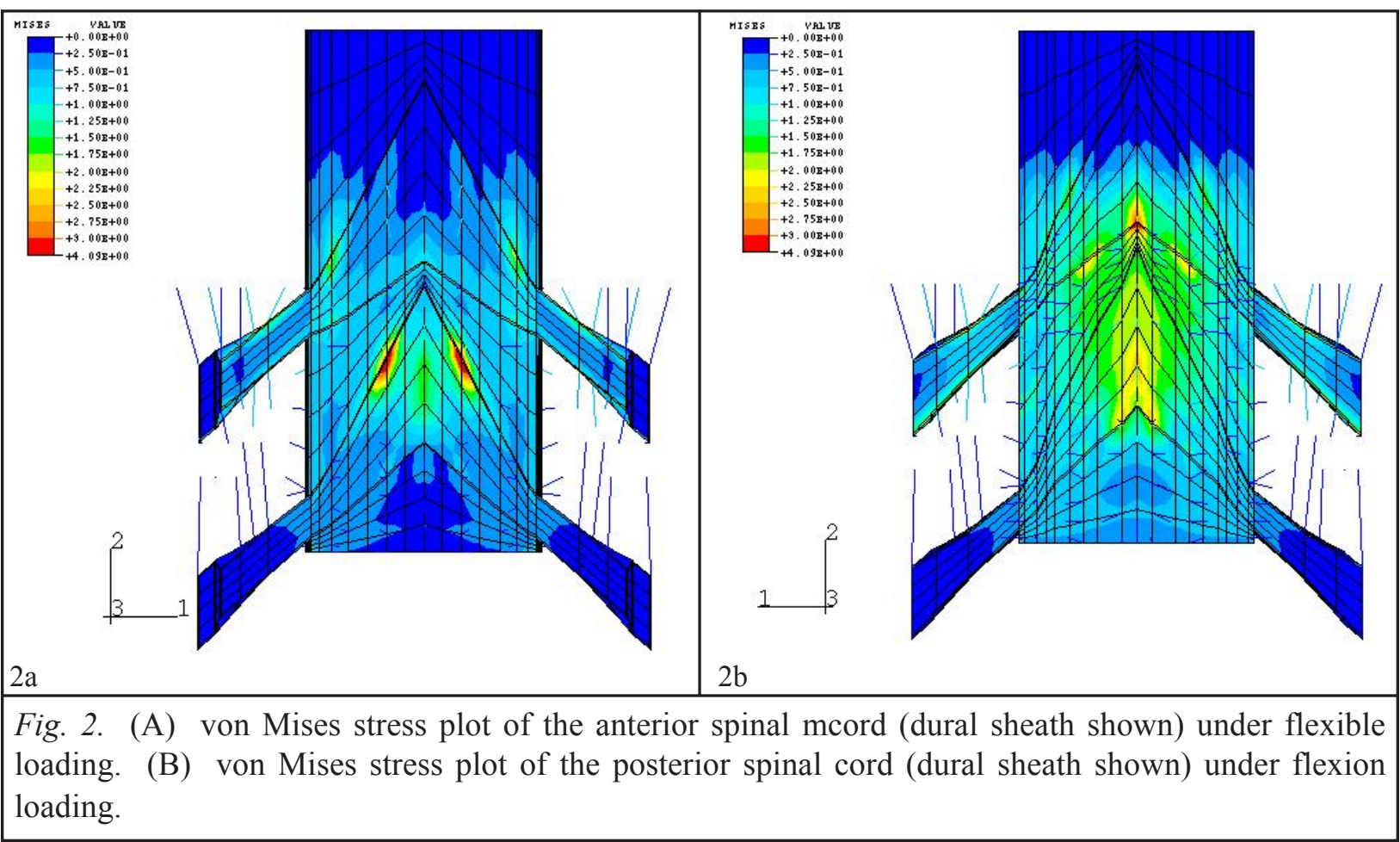


displacement and strain within the cord agree with the published data, we can attest to the correctness of the approach adapted for the development of this preliminary model. Predictions based on such an improved model will help us reach a better understanding of the mechanics of whiplash trauma and the ways in which this may be prevented and/or treated.

This FE model represents the first attempt the authors are aware of to quantify stress and strain in the spinal cord during physiologic spinal loading using a threedimensional FE cervical spine model with spinal cord. As with any analytical model, there are some limitations. First, the model was created based on a single person's CT scans so the results may not hold true for the majority of the population. On the other hand, this is one of the strengths of the model. Finite element models permit parametric studies to highlight the most important parameters. Certain assumptions about the size of the spinal cord will need to be made based on canal geometry. Secondly, the model contains no simulation of muscles or muscle forces. However, as many authors have found that muscles do not react in time to 'prevent' whiplash injury from occurring, this lack of musculature is not considered detrimental to the model with regards to the study of whiplash. Another limitation involves the inability of a quasi-static study to model a rapid injury such as that which occurs during a whiplash event. Also, since relatively little is known about the exact material properties of the cord components, assumptions have to be made based on other soft tissue or other material data in the literature. In the future, more work needs to be done to more accurately define the material properties so a better model can be generated.

The current model was tested only under the static loading conditions and thus research needs to be extended to incorporate dynamic loading conditions on the spine. Further work needs to be done to study the implications of other cervical spinal injuries such as sports related injuries, falls, and acts of violence, to determine the effects of the spinal cord to other traumatic incidents. Future studies should also include modeling the contact between the spinal cord and the vertebra, disc, and other associated structures to more accurately predict their effect on both the spine and the spinal cord. This model may also prove a useful tool in the evaluation of stresses and strains in the spinal cord as a function of changes in material properties of the spinal cord and spinal disorders such as spondylosis.

\section{REFERENCES}

1. Meyer P, Cybulski G, Rusin J, et al. Spinal Cord Injury. Neurol Clin 1991; 9:625-661

2. Koenig, G. and G. Dohrmann. Histopathological variability in "standardized" spinal cord trauma. $J$ Neurol 1997; 40:1203-1210

3. MacNab I. Acceleration injuries of the cervical spine. J Bone Joint Surg (Br). 1964; 46A:1797-1799

4. Penning, L. Acceleration injury of the cervical spine by hypertranslation of the head: Part I. Effect of normal translation of the head on the cervical spine motion: a radiological study. Eur Spine J 1992; 1:7-12

5. Penning, L. Acceleration injury of the cervical spine by hypertranslation of the head: Part II. Effect of hypertranslation of the head on the cervical spine motion: discussion of literature data. Eur Spine J 1992; 1:13-19

6. Denis, F. The three column spine and its significance in the classification of acute thoracolumber injuries. Spine 1983; 8:817-831

7. Keene, J. and S. Fischer, Significance of acute posttraumatic bony encroachment of the neural canal. Spine 1989; 14:799-802

8. Carter, J., R. Ching, A. Tencer. Transient changes in canal geometry during axial burst fracture of the cervical spine. ORS Trans 1996; 42:667

9. Ching, R., Watson, NA, Carter, JW. The effect of postinjury spinal position on canal occlusion in a cervical spine burst fracture model. Spine 1997; 22:1710-1715

10. Tencer A, Allen B, and Ferguson R. A biomechanical study of thoracolumber spinal fractures with bone in the canal. Part I: effect of laminectomy. Spine 1985; 10:580-585

11. Scifert J. Biomechanics of the Cervical Spine, Doctoral Dissertation, University of Iowa, 2000.

12. Voo L, Kumaresan S, Pintar F, et al. Finite element models of the human head. Medical \& Biological Engineering and Computing 1996; 34:375-381

13. Bilston, L. and L. Thibault. The mechanical properties of the human cervical spinal cord in vitro. Ann of Biomed Eng 1996; 24:67-74

14. Yuan Q, Dougherty L, Margulies S. In Vivo Human Cervical Spinal Cord Deformation and Displacement in Flexion. Spine 1998; 23:1677-1683

15. Van Noort R, Black M, Martin R, et al. A study of the uniaxial mechanical properties of human dura mater preserved in glycerol. Biomaterials 1981; 2:41-45

16. Onan O, Heggeness M, Hipp J. A motion analysis of the cervical spine facet joint. Spine 1998; 23:430419

17. Grauer, J, Penjabi, MM, Cholewicki J. Whiplash produces an S-shaped curvature of the neck with hyperextension at lower levels. Spine 1997; 22:24892484 
18. Clausen J. Experimental and theoretical investigation of cervical spine biomechanics - effects of injury and stabilization. Doctoral Dissertation. University of Iowa 1996.

19. Scifert J, Goel V, Smith D, et al. In vitro biomechanical comparison of a posterior plate versus facet wiring in quasi-static and cyclic modes. Proceedings of the 44th Annual Meeting of the Orthopaedic Research Society. New Orleans, 1998; 255

20. Moroney S, Schultz A, Miller J, et al. Load-displacement properties of lower cervical spine motion segments. $J$ Biomech 1988; 21:769-779 